\title{
Classification and diagnosis of congenital malformations of the female genital tract according to ESHRE/ESGE
}

\author{
Anastasios Makedos, Grigoris Grimbizis
}

1st Department of Obstetrics and Gynaecology, Aristotle University of Thessaloniki, Papageorgiou General Hospital, Thessaloniki, Greece.

Correspondence

Anastasios Makedos 3, Patriarchou loakim, 54622 Thessaloniki, Greece, email:anmakedos@yahoo.gr

\section{Abstract}

Congenital malformations of the female genital tract affect $4-7 \%$ of the female population. They are mainly detected at reproductive age during the investigation for infertility. Accurate classification is vital for the effective management. In need of a more detailed description of these anomalies, clinical usefulness and simplicity ESHRE and ESGE introduced in 2013 the new classification system and further in 2016, at the Thessaloniki consensus they introduced guidance on proper diagnosis with the use of the available diagnostic procedures. In the current review the most important key points of both consensuses are described.

Key words: ESHRE classification, ESGE classification, congenital malformations diagnosis, female genital tract.

Congenital malformations of the female genital tract, with a prevalence of $4 \%$ to $7 \%$, are defined as deviations from normal anatomy resulting from embryological maldevelopment of the Müllerian or paramesonephric ducts ${ }^{1,2,3}$. They are mainly associated with health and reproductive problems $s^{4-9}$. Due to their clinical importance and the need for more accurate approach, a reliable classification system can assist towards their management, but also make their pathogenesis more comprehensive ${ }^{10}$.

Three systems have been proposed for the classification of female genital tract anomalies: the Amer- ican Fertility Society's (AFS) currently American Society of Reproductive Medicine system ${ }^{11}$, the embryological-clinical classification system of genito-urinary malformations ${ }^{12,13}$ and the Vagina, Cervix, Uterus, Adnexae and associated Malformations system based on the Tumor, Nodes, Metastases principle in oncology ${ }^{14}$.

In need for more accurate classification, clinical usefulness and simplicity European Society of Human Reproduction and Embryology (ESHRE) and European Society for Gynaecological Endoscopy (ESGE) introduced in 2013 a new classification of fe- 
male genital anomalies with well-described classes and sub-classes ${ }^{15,16}$.

The ESHRE/ESGE classification system has the following main characteristics:

a) Systematic categorization of anomalies is based on anatomy

b) Main classes' design is based on deviations of uterine anatomy deriving from the same embryological origin

c) Main sub-classes' design is based on anatomical variations of the main classes expressing different degrees of uterine deformity and being clinically significant

d) Anomalies of the cervical and the vaginal are classified into independent supplementary subclasses.

In the classes and subclasses of the system anomalies are sorted according to increasing severity of the anatomical deviation; the less severe variants are placed at the beginning, the more deformed types at the end. For simplicity, an very detailed subclassification is avoided, and so anatomical variations of uterine, cervical and vaginal anomalies are grouped in subclasses having as a criterion the clinical significance of the abnormality.

\section{Uterine main classes and sub-classes}

Class U0 incorporates all cases with normal uterus. A normal uterus is any uterus having either straight or curved interostial line but with an internal indentation at the fundal midline not exceeding $50 \%$ of the uterine wall thickness. Thus, it was decided to define uterine deformity as proportions of uterine anatomical landmarks (e.g. uterine wall thickness). The addition of normal uterus gives the opportunity to independently classify congenital malformations of the cervix and vagina ${ }^{17,18}$.

Class U1 (Dysmorphic uterus) incorporates all cases with normal uterine outline but with an abnormal shape of the uterine cavity, excluding septa.
Class I is further subdivided into three categories;

- Class U1a (T-shaped uterus)is characterized by a narrow uterine cavity due to thickened lateral walls with a correlation $2 / 3$ uterine corpus and $1 / 3$ cervix,

- Class U1b (uterus infantilis)is characterized by a narrow uterine cavity without lateral wall thickening and an inverse correlation of $1 / 3$ uterine body and $2 / 3$ cervix

- Class U1c(others) which is added to include all minor deformities of the uterine cavity including those with an inner indentation at the fundal midline level of less than $50 \%$ of the uterine wall thickness. This aims to facilitate groups who want to study patients with minor deformities and to clearly differentiate them from patients with a septate uterus ${ }^{19}$.

Class U2 (septate uterus) incorporates all cases with normal fusion and abnormal absorption of the midline septum. Septate is defined as the uterus with normal outline and an internal indentation at the fundal midline (septum) more than $50 \%$ of the uterine wall thickness. The septum could divide partly or completely the uterine cavity including in some cases cervix and/or vagina. Class U2 is further divided into two sub-classes according to the degree of the uterine corpus deformity:

- Class U2a (partial septate uterus) is characterized by the existence of a septum dividing partly the uterine cavity above the level of the internal cervical os.

- Class U2b (complete septate uterus) is characterized by the existence of a septum fully dividing the uterine cavity up to the level of the internal cervical os. Patients of this sub-class could have or not cervical (e.g. bicervical septate uterus) and/or vaginal defects.

Class U3 (bicorporeal uterus) incorporates all cases of fusion defects. As bicorporeal is defined the uterus with an abnormal fundal outline; it is char- 
acterized by the presence of an external indentation at the fundal midline exceeding $50 \%$ of the uterine wall thickness. This indentation could divide partly or completely the uterine corpus including in some cases the cervix and/or vagina. Class U3 is further divided into three subclasses based on the degree of the uterine corpus deformity:

- Class U3a (partial bicorporeal uterus), characterized by an external fundal indentation partly dividing the uterine corpus above the level of the cervix,

- Class U3b (complete bicorporeal uterus), characterized by an external fundal indentation completely dividing the uterine corpus up to the level of the cervix

- Class U3c (bicorporeal septate uterus), characterized by the presence of an absorption defect in addition to the main fusion defect. In patients with bicorporeal septate uterus (class U3c) the width of the midline fundal indentation exceeds by $50 \%$ the uterine wall thickness.

Also, that patients with complete bicorporeal uterus (class U3b) could have or not co-existent cervical (double cervix/formerly Didelphys uterus) and/or vaginal defects (e.g. obstructing or not vaginal septum).

Class U4 (hemi-uterus) incorporates all cases of unilateral formed uterus. Hemi-uterus is defined as the unilateral uterine development; the contralateral part could be either incompletely formed or absent. Class U4 is further divided into two sub-classes depending on the presence or not of a functional rudimentary cavity;

- Class U4a (hemi-uterus with a rudimentaryfunctional cavity), characterized by the presence of a communicating or non-communicating functional con- tralateral horn

- Class U4b (hemi-uterus without rudimentaryfunctional cavity), characterized either by the presence of non-functional contralateral uterine horn or by aplasia of the contralateral part.

Class U5 (aplastic uterus) incorporates all cases of uterine aplasia ${ }^{20}$. It is a formation defect characterized by the absence of any fully or unilaterally developed uterine cavity. However, in some cases there could be bi- or unilateral rudimentary horns with a cavity, while in others there could be uterine remnants without a cavity ${ }^{21}$. Patients with aplastic uterus could usually have co-existent defects (e.g. vaginal aplasia/Mayer-Rokitansky-Küster-Hauser syndrome).

Class U5 is further divided into two sub-classesdepending on the presence or not of a functional cavity in an existent rudimentary horn;

- Class U5a (aplastic uterus with rudimentaryfunctional cavity) is characterized by the existence of bi- or unilateral functional horn.

- Class U5b (aplastic uterus without rudimentaryfunctional cavity) is characterized either by the existence of uterine remnants or by full uterine aplasia. The presence of a horn with cavity is clinically important, and it is used as a criterion for subclassification.

Class U6 incorporates unclassified cases. However, infrequent anomalies, subtle changes or combined pathologies could not be allocated correctly to one of the six groups.

\section{Co-existent cervical anomalies}

- Sub-class C0 (normal cervix) incorporates all cases of normal cervical development.

- Sub-class C1 (septate cervix) incorporates all cases of cervical absorption defects. It is characterized by the presence of a normal externally rounded cervix with the presence of a septum.

- Sub-class C2 (double cervix) incorporates all cases of cervical fusion defects. It is characterized by the presence of two distinct externally rounded cervices; these two cervices could be either fully divided or partially fused. It could be combined with a com- 
plete bicorporeal uterus as a class U3b/C2 in the formerly Didelphys uterus.

- Sub-class C3 (unilateral cervical aplasia) incorporates all cases of unilateral cervical formation. It is characterized by the unilateral, only, cervical development; the contralateral part could be either incompletely formed or absent.

- Sub-class C4 (cervical aplasia) incorporates all cases of complete cervical aplasia but also those of severe cervical formation defects. It is characterized either by the absolute absence of any cervical tissue or by the presence of severely defected cervical tissue such as cervical cord, cervical obstruction and cervical fragmentation.

\section{Co-existent vaginal anomalies}

- Sub-class V0 (normal vagina) incorporates all cases of normal vaginal development.

- Sub-class V1 (longitudinal non-obstructing vaginal septum).

- Sub-class V2 (longitudinal obstructing vaginal septum).

- Sub-class V3 (transverse vaginal septum and/or imperforated hymen).

- Sub-class V4 (vaginal aplasia) incorporates all cases of complete or partial vaginal aplasia.

Nowadays, a wide variety of non-invasive diagnostic procedures are available for the accurate diagnosis of variations of the female genital tract, with different characteristics, invasiveness and diagnostic accuracy ${ }^{22,23}$. The aim of the Thessaloniki ESHRE /ESGE consensus was to provide the researchers with recommendations for the diagnostic work-up of female genital anomalies by using the definitions of the ESHRE/ESGE classification described previously ${ }^{24}$.

The diagnostic potential but also the advantages and disadvantages of each technique will be further described.

Gynecological examination can only detect some cervical malformations (aplasia, double cervices, longitudinal septa reaching to the external cervical os) can be diagnosed objectively by inspection. Vaginal or rectal palpation cannot provide information for the uterine cavity and uterine wall and could provide only some useful, but highly subjective, information for the uterine body (e.g. complete bicorporeal uterus). Only in cases of dilatation secondary to obstruction of menstrual flow (hematocolpos/ hematometra/hemato-cavity in cases of non- communicating uterine horns) can palpation be useful. Advantages can be considered the non-invasiveness, simplicity and the low cost.It should be avoided for the diagnosis of uterine anomalies due to its inherent inability to provide reliable information for uterine anatomy and cannot be used in women who have never been sexually active before. It can be properly used in cases of primary amenorrhea, careful inspection of the external genitalia for the presence of distal vaginal aplasia. Careful inspection of the vagina, to avoid misdiagnosis in cases of longitudinal vaginal septa, by entering only in one of the two existing vaginal spaces and also careful inspection of the vaginal vault with a speculum to establish the presence of one or more cervical body(ies) or one cervical body with one or two external cervical opening(s).

X-ray hysterosalpingographycan provide useful information about the uterine cavity and the cervical canal,in the absence of cervical obstruction. It does not provide any information for the vagina (exception: blind vagina with small opening), the uterine wall, the external contour of the uterus and also for the presence of rudimentary non-communicating horns or cavities. Advantages include wide availability, additional useful information in cases of infertile women for potential intra-cavitary pathology (adhesions, polyps, myomas) and of course description of tubal morphology. Disadvantages include pain, risk of infection and irradiation of the patient. False-positive and false-negative results restrict the diagnostic 
accuracy; air bubbles can might be misleading for intra-cavity pathology; distension of the cavity due to fluid injection might distort the shape of the cavity to a degree that is related to whether there is a tubal ostia obstruction or not and, hence, limiting the value of assessing the interior contour.

Two-dimensional ultrasoundcould provide reliable, objective and measurable information not only for the anatomy of the cervix, uterine cavity, uterine wall and external contour of the uterus but also for associated pelvic pathology, e.g. ovarian pathology (e.g. benign and malignant tumors, endometriosis), hydrosalpinges, renal anomalies. Transperineal 2D ultrasound may provide information on the vaginal cavity, especially in the presence of imperforate hemivagina. Non-invasiveness, simplicity and low cost are the advantages of the technique. It offers additional valuable information in cases of infertile women for potential intra-cavitary (major adhesions might be suspected presented as "bridges" between the walls, polyps, myomas) and intramural pathology (myomas, adenomyosis). It is a dynamic examination which is highly dependent on the experience of the examiner and on the proper and systematic way of performing the procedure. In cases of vaginal obstruction or stenosis, transrectal ultrasound with vaginal or transperinealprobe is another available option to evaluate the vagina and the uterus (not in children nor in adolescents).

Hysterosalpingo-contrast sonography (HyCoSy) can provide reliable, objective andmeasurable information for the anatomy of the cervix, uterine cavity and possible defects, uterine wall, external contour of the uterus and for other peritoneal structures (e.g. ovaries) and also could be used as a tubal patency test in infertile patients. It is a simple and low-cost technique and provides more reliable information than that of 2D US, in cases of infertile women, for potential intra-cavitary (adhesions presented as "bridges" between the walls, polyps, myomas) and intramural pathology (myomas, adenomyosis) but not necessarily for uterine malformations. On the other hand, HyCoSy is a dynamic examination, which means that the findings are highly dependent on the examiner's experience on the method and the distension of the uterine cavity could potentially modify internal uterine contour resulting in false-negative imaging of the uterine cavity, especially in marginal uterine anomalies.

Three-dimensional ultrasoundcan provide highly reliable, objective, measurable information for the anatomy of the cervix, uterine cavity, uterine wall, external contour of the uterus and for relevant pelvic pathology. 3D volumes give reliable and objective representation of the examined organs more independently of the examiner overcoming the limitations of obtaining coronal images with 2D sonography. The technique is non-invasive, easily applicable to the patient, provides precise and objective measurements of the uterus and offers additional information, which is more reliable than that of 2D US, in cases of infertile women for potential intra-cavitary (adhesions the walls, polyps, myomas) and intramural pathology (myomas, adenomyosis). A transperineal 3D approach may offer the opportunity to view pelvic structures including the vagina and cervix. Diagnosis of associated vaginal anomalies can be done by transperineal acquisition of the pelvic floor volume after filling the vagina with gel or saline. Contrarily, the 3D US set-up is still not very widely available and experienced sonographers with special and adequate training in 3-dimensional image acquisition and post-processing techniques are needed.

Magnetic resonance imaging (MRI)can provide highly reliable and objective information for the anatomical status of the vagina cervix, uterine cavity, uterine wall, external contour of the uterus and for other peritoneal structures excluding the tubes. It is non-invasive, it has no radiation and gives a reliable and objective representation of the examining or- 
gans in the sagittal, transverse and coronal plane (three dimensions). It can be used for diagnosis in cases of complex and obstructing anomalies. It is more expensive and less available than ultrasound and not appropriate for patients with claustrophobia and morbid obesity. The required planes provided are limited, pre-defined and independent of the examiner, a disadvantage that could potentially impair the diagnostic accuracy of the method in the absence of an experienced radiologist.

Hysteroscopy provides highly reliable information on the anatomical status of the vagina (vaginoscopic approach), the cervical canal and, mainly, the uterine cavity and the tubal ostia. It is minimally invasive giving the additional opportunity of treating T-shaped, septate and bicorporeal septate uterus. It can also be used for evaluation of the vagina and/or cervix in case of virgo.It doesn't offer information on uterine wall thickness and uterine outline and is unable to offer differential diagnosis between septate and bicorporeal uterus.

Endoscopy, in terms of combination of laparoscopy and hysteroscopy, provides highly reliable information for the anatomical status of the vagina (vaginoscopic approach), cervical canal, uterine cavity, tubal ostia, external contour of the uterus and the intra- peritoneal structures. Visualisation of the cervical canal, the endometrial cavity and the external contour of the uterus is direct and so endoscopy is the "gold standard" for diagnosis and differential diagnosis. Endoscopic approach represents the minimally invasive route of choice in the treatment of a wide variety of female genital anomalies. Disadvantages include the invasiveness of the technique, the subjectivity of the performing clinician and of course the background of experience and training. It cannot be used as a first-line screening procedure but offers supplementary information about partial or total absence of Fallopian tubes and abnormal localization of ovaries.
Computerized tomography scanning (CTS) has no place any longer in the diagnosis of female genital anomalies due to radiation and poor depiction of the female genital structures.

\section{Discussion}

Female genital malformations begin since the embryonic stage of life and sometimes are not detected until the age when women seek for fertility. Apart though from infertility, health and psychological problems may arise due to the presence of such anomalies. The need for better understanding and more comprehensive communication between the clinicians, including the vast majority of the details in order to describe these malformations, led to the introduction in 2013 of the new classification according to ESHRE/ESGE as described previously.

Nowadays, a wide range of diagnostic tools based on medical technology are available, which sometimes can lead to a dilemma when trying to choose the appropriate diagnostic method towards accurate diagnosis. On the more recent consensus in 2016, ESHRE and ESGE provided detailed information about the available diagnostic techniques, their advantages, disadvantages and recommendations regarding their proper use.

Definitely reasonable time will be needed in order all clinicians, dealing with the investigation of female genital malformations, to start commonly using the new classification system and its recommendations. This, apart from accurate diagnosis and proper management, may lead to advantageous feedback for possible further modification of the current classification system.

\section{References}

1. Chan YY, Jayaprakasan K, Zamora J, Thornton JG, Raine-Fenning N, Coomarasamy. A The prevalence of congenital uterine anomalies in unselected and high-risk populations: a systematic 
review. Hum Reprod Update 2011;17:761-771.

2. Grimbizis GF, Camus M, Tarlatzis BC, Bontis JN, Devroey P. Clinical implications of uterine malformations and hyster- oscopic treatment results. Hum Reprod Update2001; 7:161-164.

3. Saravelos SH, Cocksedge KA, Li T-C. Prevalence and diagnosis of congenital uterine anomalies in women with reproductive failure: a critical appraisal. Hum Reprod Update 2008;14:415-419.

4. Brucker SY, Rall K, Campo R, Oppelt P, Isaacson K.Treatment of congenital malformations. Semin Reprod Med 2011;29:101-112.

5. Chan YY, Jayarpakasan K, Tan A, Thornton JG, Coomarasamy A, Raine-Fenning NJ. Reproductive outcomes in women with congenital uterine anomalies: a systematic review. Ultrasound $\mathrm{Ob}$ stet Gynecol 2011;38:371-382.

6. Fedele L, Bianchi S, Zanconato G, Berlanda N, Bergamini V. Laparoscopic removal of the cavitated noncommunicating rudimentary uterine horn: surgical aspects in 10 cases. Fertil Steril 2005;83:432-436.

7. Gergolet M, Campo R, Verdenik I, Kenda Suster N, Gordts S, Gianaroli L. No clinical relevance of the height of fundal indentation in subseptate or arcuate uterus: a prospective study. RBM Online 2012;24:576-582.

8. Grimbizis GF, Tsalikis T, Mikos T, Papadopoulos N, Tarlatzis BC, Bontis JN. Successful end-to-end cervico-cervical anastomosis in a patient with congenital cervical fragmentation: case report. Hum Reprod 2004;19:1204-1210.

9. Joki-Erkkilä MM, Heinonen PK. Presenting and long-term clinical implications and fecundity in females with obstructing vaginal malformations. J Pediatr Adolesc Gynecol 2003;16:307-312.

10. Grimbizis GF, Campo R. Congenital malformations of the female genital tract: the need for a new classification system. Fertil Steril 2010; 94:401-407.
11. American Fertility Society. The AFS classification of ad- nexal adhesions, distal tubal occlusion, tubal occlusion secondary to tubal ligation, tubal pregnancies, Mullerian anomalies and intrauterine adhesions. Fertil Steril 1988;49:944-955.

12. Acien P, Acien M, Sanchez-Ferrer M. Complex malformations of the female genital tract. New types and revision of classification. Hum Reprod 2004;19:2377-2384.

13. Acien P, Acien MI. The history of female genital tract malformation classifications and proposal of an updated system. Hum Reprod Update 2011; 17:693-705.

14. Oppelt P, Renner SP, Brucker S et al. The VCUAM (Vagina Cervix Uterus Adnex Associated Malformation) Classification: a new classification for genital malformations. Fertil Steril 2005;84: 1493-1497.

15. Grimbizis GF, Gordts G, Di Spiezio SA et al. The ESHRE/ESGE consensus on the classification of female genital tract congenital malformations. Hum Reprod 2013;28: 2032-2044.

16. Grimbizis GF, Gordts G, Di Spiezio SA et al. The ESHRE/ESGE consensus on the classification of fe- male genital tract congenital malformations. Gynecol Surg 2013;10: 199-212.

17. Rock JA, Roberts CP, Jones HW. Congenital anomalies of the uterine cervix: lessons from 30 cases managed clinically by a common protocol. Fertil Steril 2010;94:1858-1863.

18. Strawbrigde LC, Crough NS, Cutner AS, Creighton SM. Obstructive Mullerian anomalies and modern laparoscopic man- agement. J Pediatr Adolesc Gynecol 2007;20:195-200.

19. Gergolet M, Campo R, Verdenik I, Kenda Suster N, Gordts S, Gianaroli L. No clinical relevance of the height of fundal indentation in subseptate or arcuate uterus: a prospective study. RBM Online 2012;24:576-582.

20. Aittomaki K, Eroila H, Kajanoja P. A population- 
based study of the incidence of Müllerian aplasia in Finland. Fertil Steril 2001;76:624-625.

21. Oppelt PG, Lermann J, Strick R et al. Malformations in a cohort of 284 women with Mayer-Rokitansky-Ku ster-Hauser syndrome (MRKH). Reprod Biol Endocrinol 2012;10:57-64.

22. Bermejo C, Ten Martínez P, Cantarero R et al. Three-dimensional ultrasound in the diagnosis of Müllerian duct anomalies and con- cordance with magnetic resonance imaging. Ultrasound Obstet Gynecol 2010; 35(5):593-601.

23. Chan YY, Jayaprakasan K, Zamora J, Thornton JG, Raine-Fenning N, Coomarasamy A. The prevalence of congenital uterine anomalies in unselected and high-risk populations: a systematic review. Hum Reprod Update 2011;17:761-771.
24. Grimbizis GF, Di Spiezio Sardo A, Saravelos SH et al. The Thessaloniki ESHRE/ESGE consensus on diagnosis of female genital anomalies.Gynecol Surg 2016;13:1-16.
Received 5-4-2018

Revised 10-6-2018

Accepted 22-6-2018 